\title{
TOD UND ZEIT IM ALTEN ÄGYPTEN'
}

Die ägyptischen Pyramiden nannte Muhammad al-Maqrizi, ein arabischer Gelehrter des Mittelalters, „Bauten, vor denen sogar die Zeit sich fürchtet, wo doch alles in der Welt fürchtet die Zeit“. Sie sollen uns als Einstieg dienen in unser Thema ,Tod und Zeit im Alten Ägypten'. Die Pyramiden sind gegen die Zeit gebaut, das hat al-Maqrizi klar gesehen und brillant formuliert; und sie sind auch gegen den Tod gebaut: Sie sind Gräber, die der Unsterblichkeit der in ihnen bestatteten Pharaonen dienen. Sie veranschaulichen unser Thema ,Tod und Zeit‘ im Modus der Negation, indem sie beides leugnen. Sie verneinen den Tod, aber sie verdrängen ihn nicht, ganz im Gegenteil. Sie geben ihm - im Modus der Negation - eine überwältigende Sichtbarkeit. Das müssen wir schon einmal als erstes Charakteristikum des altägyptischen Umgangs mit dem Tod festhalten.

Da der Mensch das einzige Lebewesen ist, das um seine Vergänglichkeit weiß, ist sein Zeitbewusstsein - vor allem in vormodernen Gesellschaften vom Tod her bestimmt. Die Zeit gliedert sich ihm in vergängliche Lebensund unvergängliche Todeszeit, die man sich entweder in einer Todeswelt (Hades) oder in einer elysischen Welt ewigen Lebens (Paradies) vorstellt. Die Lebens- oder Oberwelt ist die Welt, in der die Lebenden leben, und die Unteroder Todeswelt ist die Welt, in der die Toten tot sind. Dass die Toten weiter existieren und nicht einfach aus der Welt verschwinden, ändert nichts daran, dass sie nicht etwa weiterleben, sondern tot sind. Es gehört zur archaischen Definition des Totseins, dass man aus der Lebenswelt nicht einfach ins Nichts verschwindet, sondern in die Todeswelt als dem Ort der Toten übergeht. Diesen Ort nennen die Griechen Hades, die Hebräer Scheol, die Babylonier das ,Land ohne Wiederkehr' und die Römer den Orcus. Aus der Todeswelt gibt es normalerweise keine Errettung, weder zurück in die Lebenswelt, wie es Orpheus für Eurydike versucht und Jesus für Lazarus bewirkt hat, noch in die Götterwelt, was allenfalls einmal als eine so große und seltene Ausnahme geschieht, dass sie durch ihre Exzeptionalität die Regel bestätigt. Die Menschen existieren als Sterbliche nacheinander in zwei Welten, der Lebenswelt und der Todeswelt.

Unveränderter Abdruck des am 6. Dezember 2012 in Weimar gehaltenen Vortrags. Auf Fußnoten habe ich verzichtet; die interessierte Leserin findet alle Einzelheiten in meinen Büchern Tod und Jenseits im Alten Ägypten, 2. Aufl., München, 2003, und Steinzeit und Sternzeit. Altägyptische Zeitkonzepte, München, 2011. 
Die Pyramide aber bezieht sich nicht auf die Todeswelt, sondern auf die Götterwelt, auf den Himmel als ein Elysium, in dem der König vom Tod befreit und als Gott unter die Götter aufgenommen ist. Vom König nahm man an, dass er als ein Gott auf Erden herrschte, nach dem Tode zum Himmel aufflog und sich mit dem Sonnengott, seinem himmlischen Vater, vereinigte. Dieser Glaube findet in der Bauform der Pyramide seinen Ausdruck. Die Pyramide symbolisiert - und bewirkt dadurch nach dem Glauben der Menschen - mit ihrer zum Himmel weisenden Spitze und ihrer genauen Ausrichtung nach den Himmelsrichtungen den Himmelsaufstieg des toten Königs und seine Eingliederung sowohl in den Sonnenlauf als auch in die Zirkumpolarsterne am nächtlichen Nordhimmel, die als einzige während der Nacht nicht untergehen. Eben darauf verweisen auch die vielen Texte, die seit Unas, dem letzten König der fünften Dynastie (um 2350 v. Chr.), in ihren inneren Kammern aufgezeichnet sind. Der Himmelsaufstieg des verstorbenen Königs ist ihr zentrales Thema. Die Pyramide hält auf diese Weise Himmel und Erde, Jenseits und Diesseits zusammen, und genau das ist es, worum es in der ägyptischen Kultur von allem Anfang an und vor allen Dingen geht. Der tote König ist der Garant dieses Zusammenhangs, daher ist er der ,große oder geradezu ,der größte` Gott - der ägyptische Ausdruck lässt beide Übersetzungen $\mathrm{zu}-$, und alle kulturellen und insbesondere baulichen Anstrengungen des Landes gelten seiner Verehrung.

Mit der Figur des Königs kommt eine Zweiteilung von Tod und Zeit in die ägyptische Welt - und im Lauf der Zeit wohl auch darüber hinaus: in Tod und Unsterblichkeit sowie Zeit und Ewigkeit. Im dritten Jahrtausend, dem ,Alten Reich', in dem die Bauform der Pyramide entwickelt wurde und ihre höchste Vollendung erreichte, war das Elysium dem König vorbehalten. Er schwang sich im Tode falkengleich himmelan, während die Menschen sich, wie es heißt, in der Erde verbergen. In den Pyramidentexten bildet die Distanzierung des Königs vom Schicksal alles Sterblichen das zentrale Thema. Das Elysium war also eine im Ursprung politische Idee; es überragte so weit die Todeswelt, wie die Gestalt des Königs die Menschenwelt und wie die Pyramide die Gräber der anderen überragte. Der König verkörpert die Negation der Zeit und des Todes. Er stirbt nicht und kennt weder Vergehen noch Aufhören. Seine Zeit ist das Immer-wieder und das unwandelbare Bleiben - die Negation der Zeit.

Für die Zeit im Modus der Negation, die Nicht-Zeit, haben wir im Deutschen das Wort ,Ewigkeit‘. Die Ägypter haben dafür zwei Worte, von denen sich das eine, Neheh, auf die Unaufhörlichkeit des Immer-wieder und das andere, Djet, auf die Unendlichkeit der unwandelbaren Dauer bezieht. Hinter dieser Unterscheidung steht die Aspektopposition der semitischen Sprachen. So, wie die uns vertrauten Sprachen zwischen den Zeitstufen Vergangenheit, Gegenwart und Zukunft unterscheiden, auf denen unser Zeitbegriff seit Platon und Augustinus aufbaut, so unterscheiden die semitischen Sprachen zwischen den Aspekten Perfektiv und Imperfektiv, abgeschlossenen und unabgeschlos- 
senen Prozessen, Zeit von innen und Zeit von außen gesehen und bauen auf dieser Opposition ihren Doppelbegriff der Ewigkeit auf. Neheh ist die Zeit, die wir immer nur aus dem Inneren ihres Verlaufs heraus in den Blick fassen können, die sich in der Abfolge der Stunden, Tage und Jahre unabsehbar erneuert und wiederholt. Djet dagegen ist die Zeit, in der alles Vollendete, Abgeschlossene, Perfekte in unwandelbarer Dauer aufgehoben ist. Die beiden Zeitbegriffe haben sehr viel mit der ägyptischen Todeserfahrung zu tun. Beide Begriffe negieren den Tod, der eine im Sinne des Aufhörens und Nie-wieder, der andere im Sinne des Vergehens und Verschwindens. So kann man vom König sagen:

„Die Lebenszeit des Unas ist Neheh, die Grenzen des Unas sind Djet."

Das kann man nur im Sinne einer Negation umschreiben:

„Seine Lebenszeit hat kein Ende, und seine Existenz kennt keine Grenzen."

Indem die Begriffe Neheh und Djet die menschlichen Vorstellungen von Tod und Zeit negieren, beziehen sie sich auf ein Konzept von Transzendenz und gewinnen eine religiöse Bedeutung, die ja auch unserem Begriff von Ewigkeit eignet. Beide ägyptischen Ewigkeitsbegriffe oder Zeitnegationen sind untrennbar verbunden mit den beiden höchsten ägyptischen Göttern, mit denen das Jenseitsschicksal des Königs in Verbindung steht. Nach dem Tod vereinigt sich der König mit Re und wird zu Osiris.

Re, der Sonnengott, ist der Exponent der Neheh-Zeit und der zyklischen Zeitauffassung, der Zeit des unabsehbaren Immer-wieder, der unaufhörlichen Erneuerung und ewigen Wiederkehr. Das ist der Gott, mit dem sich der König nach dem Tod vereinigen möchte. Um das zu erreichen, darf er nicht unwandelbar fortdauern, sondern muss sich verwandeln. In allen möglichen Gestalten will er zum Himmel aufsteigen. Der Wunsch nach Verwandlung gehört ganz zur Neheh-Zeit der Erneuerung; das ägyptische Wort cheper (,werden, sich verwandeln"), geschrieben mit dem Skarabäus als Symbol dieser unaufhörlichen Kraft zur Regeneration, bezeichnet präzise die Seinsform im Rahmen des Neheh, des Immer-wieder. Anders als im griechischen Denken, dem das Sein alles und das Werden nichts bedeutete, ist im ägyptischen Denken das Werden der zentrale Heilsbegriff. Dem steht das Verbum wenen (,sein, existieren, währen, fortdauern") gegenüber. Dieses Verbum verbindet sich ebenso mit Osiris, wie das Verb cheper zu Re gehört. Re heißt in seinem Aspekt der erneuernden Verwandlung als Morgensonne Chepre (,der Werdende"), und so heißt Osiris in seinem Aspekt der unwandelbaren Fortdauer als Mumie Wan-nafre (griech. Onnophris, "der in Vollendung Währende“). Osiris ist der Exponent der Djet-Zeit, in der ein zu seiner vollendeten Gestalt ausgereiftes Leben unwandelbar aufgehoben fortdauert. Neheh, die Zeit des 
Re, ist die Zeit der Erneuerung; Djet, die Zeit des Osiris, ist die Zeit der Erinnerung.

Mit dem Untergang des Alten Reichs weitete sich das königliche Jenseitsbild auf alle Menschen aus. Damit verlor die Unterscheidung zwischen Todeswelt und Elysium ihren politischen Sinn (dem König das Elysium, den Menschen die Todeswelt) und öffnete sich anderen Interpretationen. Sie wurde vor allem zu einer Frage der Moral. So kam es zur Idee des Totengerichts. Nicht die Göttlichkeit des Königsamtes errettete den Toten aus der Todeswelt, sondern seine Rechtfertigung vor dem Tribunal der 42 göttlichen Richter und seinem Vorsitzenden Osiris. Diese Rechtfertigung wird jetzt als Vorbedingung nicht nur zur Fortdauer im sozialen Gedächtnis, also auf Erden, verstanden, sondern auch zum Übergang in die elysische Welt, in der man von Tod und Vergessen erlöst ist. Ich übergehe den gefahrvollen Weg, den die Toten gleich nach ihrem Tod durch die 21 Tore der Unterwelt zurücklegen, und das Verhör, dem sie sich vor Betreten der Gerichtshalle unterziehen mussten, und wende mich gleich dem Verfahren in der Gerichtshalle zu. Hier haben die Verstorbenen - nach Begrüßung der Richter und des Vorsitzenden, in denen sie sich bereits als unschuldiger, moralisch vollkommener Mensch darstellen - eine Liste von 82 Sünden aufzusagen mit der Beteuerung, sie nicht begangen zu haben. Währenddessen - so stellt es das zugehörige Bild dar - wird ihr Herz auf eine Waage gelegt und gegen ein Symbol der Ma'at (Wahrheit/Gerechtigkeit) abgewogen. Dabei kommt es darauf an, dass das Herz leichter als dieses Symbol - eine Feder - bleibt. Bei jeder Lüge würde die Waagschale mit dem Herzen sinken. Dieses Bild vom Totengericht mit der Waage lebt in der christlichen Ikonografie weiter, wo es in die Vorstellung vom Jüngsten Gericht übernommen wird. Die gerechtfertigten Toten aber gehen in das ägyptische Elysium ein, das ,Binsengefilde', wo man den Göttern nahe, mit Nahrung versorgt und für immer vom Tod erlöst ist.

Der ägyptische Mensch möchte in beiden Ewigkeiten Zeit und Tod überwinden. Er ist auf Re wie auf Osiris angewiesen. Er möchte als Ba-Seele sich verwandeln und im Neheh kreisen wie Re, und er möchte als Mumie im Steinernen dauern wie Osiris, in unwandelbarer Vollendung. So heißt es zum Beispiel im ägyptischen Balsamierungsritual:
„Möge dein Ba existieren, indem er im Neheh lebt
wie Orion im Leib der Himmelsgöttin;
und indem dein Leichnam dauert in der Djet wie der Stein des Gebirges."

Für dieses Ziel der osirianischen Fortdauer braucht er die Einbalsamierung, die Mumifizierung und, vor allem, das Totengericht. Die Idee der Vollendung, ägyptisch $n f r w$, hat nicht nur die Konnotationen der Schönheit, Vollkommenheit und Unvergänglichkeit, sondern auch und vor allem die der Tugend und Gerechtigkeit, der moralischen Perfektion und der Entsprechung zu den Normen der Gerechtigkeit (Ma'at). Wer sein Leben nach den Normen der Ma'at 
geführt hat, den wird sie nach dem Tod bei der Hand nehmen und gemeinsam mit ihm ins Jenseits übergehen.
„Die Ma'at aber wird ewig sein.
Sie steigt an der Hand dessen, der sie übte, ins Totenreich hinab.
Er wird begraben und vereint sich der Erde;
sein Name aber wird nicht ausgelöscht werden auf Erden, sondern man gedenkt seiner wegen der Tugend."
(Klagen des Bauern)

Daraus erwächst der Djet-Zeit eine moralische Perspektive. Nur das Gute vermag unwandelbar fortzudauern, das Böse, Schlechte, Unreine und Unvollkommene ist der Vergänglichkeit preisgegeben. Über die Unvergänglichkeit eines Resultats entscheidet seine moralische Qualität, das heißt seine Entsprechung zur Ma'at. Durch die Idee des Totengerichts, die in Ägypten um 2000 v. Chr. aufkommt, erhält die Beziehung von Tod und Zeit eine moralische Bedeutung. Im Licht dieser Idee erscheint der sterbliche, aber nach Unsterblichkeit strebende Mensch als sündiges, der Rechtfertigung bedürftiges Wesen. Durch Tod und Totengericht erscheint die Lebenszeit im Modus der Resultativität. Die verbrachte Zeit hat zu etwas geführt, ein Resultat gezeitigt, das im Totengericht gewogen, zur Vollendung gebracht und auf Dauer gestellt wird.
„Verlasse dich nicht auf die Länge der Jahre!
Sie sehen die Lebenszeit als eine Stunde an.
Wenn der Mensch übrig bleibt nach dem Landen,
werden seine Taten als Summe neben ihn gelegt."

So heißt es in der Lehre für Merikare. In diesen Versen wird die Verbindung gezogen zwischen der Kategorie der Resultativität - der ,Summe der Taten “ und der Zeit des Menschen. Die Totenrichter sehen diese Zeit als ,eine Stunde' an, im Modus des Gewesenseins, als ein abgeschlossenes Ganzes, und der verantwortlich Lebende muss sich diese Sicht des Lebens jederzeit zu eigen machen können, anstatt sich in der Befangenheit der Innensicht der Illusion endloser Fortsetzung hinzugeben. Wer auf die Länge der Jahre vertraut, fällt der Vergänglichkeit anheim. Verantwortlich leben heißt, sich an den ,Gesetzen der Ma' ${ }^{\prime} t^{`}$ zu orientieren.

„Solange etwas ist, ist es nicht das, was es gewesen sein wird.“ Dieser Satz, mit dem Martin Walsers Roman Der springende Brunnen beginnt, führt uns unmittelbar einleuchtend den Unterschied vor Augen, den es bedeutet, ob wir mitten in einem Geschehen befangen sind oder von außen darauf zurückblicken. Oft nehmen die Dinge erst in der Rückschau die Konturen einer sinnvollen Geschichte an, die man verstehen, erzählen und erinnern kann. Das Leben wird vorwärts gelebt, aber rückwärts verstanden, soll Kierkegaard sinngemäß gesagt haben. Erst vom Ende her werden die einzelnen Ereignisse lesbar. Das Ende, die Frage, ,ob etwas dabei herausgekommen ist', entscheidet über Sinn und Zusammenhang des Geschehenden. Nicht dass wir, solange 
wir noch mitten in einer Geschichte drinstehen, im Dunkeln tappen würden. Keineswegs, auch hier herrscht ein mehr oder weniger helles Licht, das von unseren Sinnvorgaben, Zielen, Erwartungen, Erinnerungen und intuitiven Orientierungen ausgeht. Aber diese Beleuchtung ist eine grundsätzlich andere und lässt die Dinge anders erscheinen als das klare Licht, das vom Ende ausgeht.

Wir sind naturgemäß unfähig dazu, unser Leben unter dem Blickwinkel des Gewesenseins ins Auge zu fassen. Genau diesen Blick aber hat sich der Ägypter gewissermaßen antrainiert, indem er, sobald es ihm seine Einkünfte und seine Stellung erlaubten, daran ging, sich ein Grab anzulegen und es mit seiner Lebensgeschichte $\mathrm{zu}$ beschriften. Die Institution des ägyptischen Monumentalgrabes mit allem, was dazugehört, von der Mumifizierung des Leichnams bis zur biografischen Inschrift, beruht auf der Grundlage der Resultativität. Dieses einzigartige Phänomen versteht man in seiner überragenden Kulturbedeutung für die ägyptische Welt nur, wenn man sich klarmacht, welche Rolle die Kategorie der Resultativität im ägyptischen Denken spielt und wie zentral sie in der ägyptischen Sprache verankert ist. Resultativität setzt zweierlei voraus: eine lineare Zeitauffassung und einen Begriff des Endes, der dem linearen Zeitverlauf ein Ziel setzt. Anders als in der zyklischen Zeitauffassung geht das Ende hier nicht über in beziehungsweise wird aufgehoben durch einen neuen Anfang, sondern es bleibt als Ende bestehen in der Form des Resultats, und alle kulturellen Anstrengungen richten sich in dieser Perspektive darauf, das Resultat zu bewahren.

„Seit ich mein Grab sah, will ich nichts, als leben“, ruft der Prinz von Homburg aus, ,und frage nichts mehr, ob es rühmlich sei!“ Der Anblick seines eigenen frisch ausgehobenen Grabes hat ihm einen tiefen Schock versetzt. Der Ägypter hingegen hat einen großen Teil seines Lebens im Anblick seines Grabes geführt. Mehr noch: Die Anlage seines Grabes war das wichtigste Projekt seines Lebens, das über viele Jahre hin sein Sinnen und Trachten in Anspruch nahm. Das Grab symbolisierte für ihn alle seine Hoffnungen auf Unvergänglichkeit. Es stellte ihm aber, ganz im Gegensatz zu der Erfahrung des Prinzen von Homburg, auch die Frage nach der Rühmlichkeit seines Lebens vor Augen. Als er sein Grab sah, so könnte man in Anlehnung an Kleists Verse sagen, wurde ihm bewusst, dass alles darauf ankommt, ein rühmliches Leben zu führen oder besser: geführt zu haben. In Gestalt seines Grabes stellte er sich einen Spiegel vor Augen, der ihm sein Leben im Licht des Gewesenseins zeigte. In diesem Spiegel erblickte er sich nicht, wie er war, sondern wie er gewesen sein wollte, in einer Endgestalt, die ihre normativen Ansprüche an seine Lebensführung stellte. Im Grab begegnete er seinem eigenen Gewesensein, der Endgestalt seines Lebens, seinem Doppelgänger, den er auf den Wandbildern darstellte und dem die Inschriften eine unvergängliche Stimme gaben, so, wie er hoffte, im Falle eines seligen Todes im Jenseits seinem , $\mathrm{Ka}^{‘} \mathrm{zu}$ begegnen. Das Grab war für den Ägypter das Instrument einer normativen Selbstverdopplung. In den Texten und Darstellungen konnte er im 
Vorgriff auf den Tod sein Leben in der Endgestalt zeigen, in der es der Vergänglichkeit und dem Vergessen enthoben für immer unwandelbar in jener Zeit oder Ewigkeit fortzudauern befähigt war, die er Djet nannte.

Die Idee des Totengerichts mit ihrer Betonung der Resultativität hat das ägyptische Zeitbewusstsein auf eine sehr grundlegende Weise geprägt. Das Gedicht aus der Lehre für Merikare, von dem ich die Strophe zitiert habe, in der von der Länge der Jahre die Rede ist, die vor den Totenrichtern zu einer Stunde zusammenschrumpft, stellt dieser Stunde die Ewigkeit im Jenseits gegenüber:

„Das Dortsein aber währt ewig.

Ein Tor, wer tut, was sie tadeln.

Wer zu ihnen gelangt ohne Frevel, der wird dort sein als ein Gott, frei schreitend wie die Herren der Ewigkeit.“

Ein Text im Grab des Wesirs Amun-User aus dem 15. Jahrhundert v. Chr. bringt das resultative Denken der Ägypter, das ihrer gesamten Grabkultur zugrunde liegt, in unübertrefflicher Klarheit zum Ausdruck:

„Ich errichtete mir ein vortreffliches Grab

in meiner Stadt der Zeitfülle.

Ich stattete vorzüglich aus den Ort meiner Felsgrabanlage

in der Wüste der Ewigkeit.

Möge mein Name dauern auf ihm

im Munde der Lebenden,

indem die Erinnerung an mich gut ist bei den Menschen

nach den Jahren, die kommen werden.

Ein Weniges nur an Leben ist das Diesseits, die Ewigkeit [aber] ist im Totenreich.“

Diesen Zusammenhang zwischen Grabbaukunst und resultativem Denken, jenem semantischen Komplex aus Gerechtigkeit, Gedächtnis und Unvergänglichkeit, hat noch über 1000 Jahre später ein in Ägypten lebender Grieche mit einer unvergleichlichen Präzision beschrieben, und man kann sich nur wundern, wie es möglich ist, dass Hekataios von Abdera eine Vorstellungswelt so getreulich reproduzieren kann, die sich 2000 Jahre zuvor entwickelt hat. Die unglaubliche Langlebigkeit dieser Gedanken zeigt, dass wir uns hier in der Mitte, im innersten Kern des kulturellen Gedächtnisses der Ägypter befinden, dort, wo die Kräfte der Bewahrung, Befestigung und Kanonisierung am intensivsten greifen. Das zu Lebzeiten angelegte Monumentalgrab ist gewiss die eindrucksvollste und intensivste Form, in der in Ägypten der Tod in das Leben der Menschen hineingewirkt und ihr Zeitbewusstsein bestimmt hat.

Hekataios von Abdera lebte zu Beginn der Ptolemäerzeit, von 320 bis 305 v. Chr., in Alexandria und schrieb ein großes, nur in Zitaten erhaltenes Werk über Ägypten. Darin heißt es: 
„Die Einheimischen geben der im Leben verbrachten Zeit einen ganz geringen Wert. Dagegen legen sie das größte Gewicht auf die Zeit nach ihrem Tode, während der man durch die Erinnerung an die Tugend im Gedächtnis bewahrt wird. Die Behausungen der Lebenden nennen sie ,Absteigen“ [katalyseis], da wir nur kurze Zeit in ihnen wohnten. Die Gräber der Verstorbenen bezeichnen sie als ,ewige Häuser' [aidioi oikoi], da sie die unendliche Zeit im Hades verbrächten. Entsprechend verwenden sie wenig Gedanken auf die Ausrüstung ihrer Häuser, wohingegen ihnen für die Gräber kein Aufwand zu hoch erscheint.“

Hekataios war aufgefallen, dass die Ägypter ihre Wohnhäuser aus luftgetrockneten Lehmziegeln bauen, das billigste und schlichteste Baumaterial, das sich denken lässt. Auch der Königspalast ist aus diesem Material errichtet. Dagegen sind die Gräber aus Stein gebaut, entweder wie kleine Tempel aus behauenen Blöcken oder aus dem Fels herausgehauen. Das hat, wie er meint, seinen Grund in der ägyptischen Konstruktion der Zeit, und diese wiederum erwächst aus den ägyptischen Begriffen von Tod und Unsterblichkeit. Das Wissen um die Sterblichkeit des Menschen lässt die Lebenszeit für den Ägypter zu einem kurzen Augenblick zusammenschrumpfen, für den es nicht lohnt, sich auf Erden aufwendig einzurichten. Dafür investieren sie alle geistigen und materiellen Mittel in die Ewigkeit oder besser unendlich lange Zeit, während derer sie nach ihrem Tod wegen ihrer Tugend im Gedächtnis bewahrt bleiben. Hekataios identifiziert genau wie Amen-User 1100 Jahre vor ihm die Unsterblichkeit mit dem Erinnertwerden aufgrund der Tugend. Die eigentlichen Investitionen gelten dem Gedächtnis; das Grab ist nur das Zeichen, der Außenhalt der Erinnerung, die sich an ein in Tugend und Gerechtigkeit geführtes Leben heftet.

Nun ist das ägyptische Monumentalgrab - als Medium der Verewigung des Gewesenseins, des unwandelbar fortdauernden Resultats eines im Sinne der $M a$ 'at geführten Lebens - nicht nur ein Phänomen des Steinernen, sondern auch ein literarisches Phänomen. Auf zwei Wegen und in zwei Formen ist die Schrift in das ägyptische Grab hineingekommen. Der erste Schritt wurde schon früh getan, um $3000 \mathrm{v}$. Chr., in der Gründungsphase des ägyptischen Reiches, als die Schrift erfunden wurde. Da entstand bereits die Sitte, das Grab des Verstorbenen mit einer Stele zu kennzeichnen, die seinen Namen und Titel enthält. Das ist nichts Besonderes; so etwas gibt es in vielen Kulturen und ist bei uns bis heute üblich. In Ägypten aber war dies der Keim für eine große Tradition und Literatur ,sepulkraler Selbstthematisierung', die in mancher Hinsicht auf die entstehende ägyptische Literatur eingewirkt hat. Eines der beliebtesten und berühmtesten Literaturwerke des Alten Ägypten, die Erzählung des Sinuhe, orientiert sich an diesem Vorbild und gibt sich als Abschrift einer solchen Grabinschrift. Der ägyptische Sinuhe-Roman verhält sich also zur Gattung der elaborierten biografischen Grabinschrift ebenso wie zum Beispiel der Roman Robinson Crusoe von Daniel Defoe zur Gattung des authentischen Reiseberichts. 
Die enge Beziehung zwischen Grab und Schrift lässt sich noch weiter vertiefen. Seine Stellung in der ägyptischen Welt kann man nur mit unseren Vorstellungen von Kunst, Autorschaft und Werk vergleichen. Dieser Vergleich scheint weit hergeholt, aber er findet sich - wie man weiß - schon bei Horaz, der sein Odenbuch mit den Pyramiden vergleicht, und auch Horaz steht damit in einer letztlich auf Ägypten selbst zurückgehenden literarischen Tradition, denn in einem Weisheitsbuch aus dem 13. Jahrhundert v. Chr. lesen wir von den großen Klassikern der Vergangenheit:

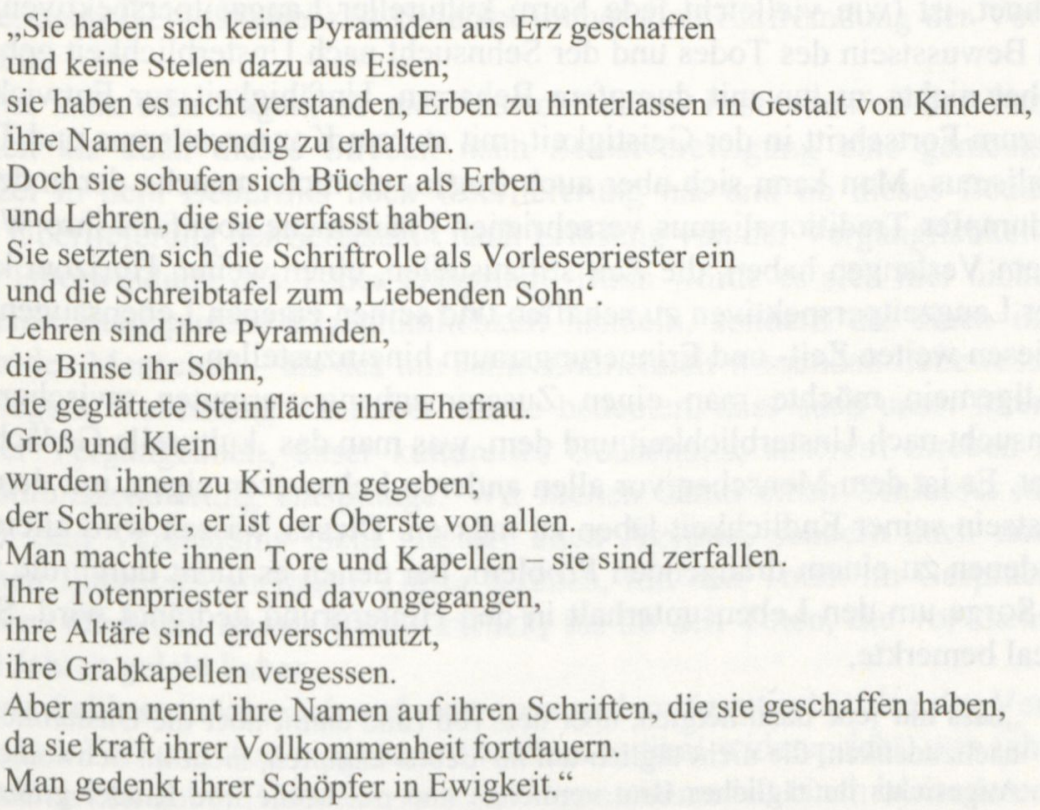

Die Literatur erscheint hier als die Fortsetzung oder vielmehr Überbietung der Monumentalarchitektur, des ,Steinernen', mit anderen, geistigen Mitteln. Der entscheidende gemeinsame Nenner aber zwischen Grab und Buch bildet die Kategorie der Autorschaft. Sie dürfte in anderen Kulturen keine Parallele haben. Wo sonst tritt der Grabherr als ,Autor' seines Grabes und seines darin aufgezeichneten Lebens in Erscheinung? Gräber werden von den Hinterbliebenen errichtet, unter Umständen aufgrund langfristiger Vorbereitung und Veranlassung des Verstorbenen. Sie sind aber kaum als Organe einer umfassenden sprachlichen und bildlichen Selbstthematisierung zu verstehen. Hier liegt das besondere, ,literarische ' Element der ägyptischen Monumentalgräber. Kunst, Literatur und Grab haben eine gemeinsame Wurzel im resultativen Denken: in der Idee, ein Resultat zu schaffen, das in der Erinnerung der Menschen fortdauert und so zum Medium nicht nur der Selbstthematisierung, sondern auch der Selbsttranszendierung und Selbstverewigung werden kann.

Solche Fortdauer basiert nicht nur auf der Vollkommenheit des Resultats, sondern auch auf dem kulturellen Gedächtnis als Prinzip einer diachronen 
kulturellen Resonanz und Transparenz. Die Texte müssen lesbar bleiben, auch nach Tausenden von Jahren. Das kulturelle Gedächtnis muss Langzeitperspektiven ausbilden und stabilisieren, in denen die Toten weiterleben und ein Gespräch über die Jahrhunderte, vielleicht gar Jahrtausende möglich wird. Schrift und Aufschreibesysteme sind nötig, aber auch Formen einer Institutionalisierung von Permanenz, die Wandel stillstellt, das Alte heiligt, Rückgriffe und Rückwärtsorientierungen favorisiert und ein Fremdwerden der Vergangenheit verhindert. Die stabile Transparenz, die die ägyptische Kultur auszeichnet, ist (wie vielleicht jede Form kultureller Langzeitperspektiven) aus dem Bewusstsein des Todes und der Sehnsucht nach Unsterblichkeit geboren. Sie hat nichts zu tun mit dumpfem Beharren, Unfähigkeit zur Entwicklung und zum Fortschritt in der Geistigkeit, mit sturem Konservatismus und Traditionalismus. Man kann sich aber auch fragen, ob nicht manche der anderswo als dumpfer Traditionalismus verschrienen Phänomene ebenfalls ihre Wurzel in dem Verlangen haben, die Zeit stillzustellen, einen weiten Horizont kultureller Langzeitperspektiven zu schaffen und seinen eigenen Lebensaugenblick in diesen weiten Zeit- und Erinnerungsraum hineinzustellen.

Allgemein möchte man einen Zusammenhang vermuten zwischen der Sehnsucht nach Unsterblichkeit und dem, was man das ,kulturelle Gedächtnis nennt. Es ist dem Menschen vor allen anderen Lebewesen eigen, mit dem Bewusstsein seiner Endlichkeit leben zu müssen. Dieses Wissen wird allerdings nur denen zu einem drängenden Problem, bei denen es nicht durch die tägliche Sorge um den Lebensunterhalt in den Hintergrund gedrängt wird. Schon Pascal bemerkte,

„dass nur jene dazu neigten, über den Tod (und damit über die Unsterblichkeit) nachzudenken, die nicht täglich um ihr Leben kämpfen, nicht im Schweiße ihres Angesichts ihr tägliches Brot verdienen und mit Mühe ihre Kinder großziehen mussten."

Das betrifft denselben Personenkreis, der „,von dreitausend Jahren sich weiß Rechenschaft zu geben“ und nicht dazu verurteilt ist, „im Dunkeln unerfahren“ zu bleiben und „von Tag zu Tag zu leben“ (wie Goethe im Buch des Unmuts seines West-Östlichen Diwan das Ideal des kulturellen Gedächtnisses umrissen hat). Die Sorge um Fortdauer und Unsterblichkeit sind ebenso wie die Pflege des kulturellen Gedächtnisses elitäre Geschäfte, aber sie hängen darüber hinaus noch sehr viel enger zusammen. Das kulturelle Gedächtnis scheint mir die wichtigste jener „Lebensstrategien“ darzustellen, die Zygmunt Baumann in seinem Buch über Tod und Unsterblichkeit herausgestellt hat.

In einem solchen Jahrtausende umfassenden Erinnerungsraum lebten die Ägypter, denen Hekataios begegnete, um sie über den Sinn ihrer eigentümlichen Sitte zu befragen, in Lehmhütten zu wohnen und sich in steinernen Palästen begraben zu lassen, und die sich so hervorragend unterrichtet zeigten über die jahrtausendealte mit dieser Praxis verbundene Semantik. Sie blickten in eine Vergangenheit zurück, deren Tiefe die Griechen als märchenhaft an- 
muten musste, ging sie doch weit über jene Grenze hinaus, an der ihre eigenen Stammbäume, falls sie von Adel waren, in die Heroen- und Götterwelt überzugehen pflegten. Die Ägypter jener Zeit mussten von einem ganz besonderen Vergangenheitssinn und -bewusstsein durchdrungen gewesen sein, das seine Grundlage in der Grabkultur hatte und sich damit wiederum vom Tode ableitete. Das Besondere dieses Vergangenheitsbewusstseins war, dass diese Vergangenheit nicht fremd geworden war im Laufe der langen Zeit. Daher ist dieses Vergangenheitsbewusstsein kein Geschichtsbewusstsein im engeren Sinne, denn Geschichtlichkeit bedeutet immer eine Entfremdung der Vergangenheit.

Man kann sich fragen, ob nicht sowohl dieses Festhalten an der Vergangenheit als auch dieses Streben nach Selbstverewigung eine gemeinsame Wurzel in dem Bedürfnis nach Überlieferung hat und ob dieses Bedürfnis nach Überlieferung der Sehnsucht nach Erlösung von der Vergänglichkeit und nach Überwindung des Todes entspringt. Dann würde es sich hier nicht nur um eine altägyptische Eigentümlichkeit handeln, sondern um einen in der Natur des Menschen - als des um seine Endlichkeit wissenden Lebewesens angelegten Wesenszug. Das aber würde bedeuten, dass auch unser Interesse an der Vergangenheit, unser kulturelles Gedächtnis, unserem Streben nach Selbsttranszendierung entspränge. Wir hielten damit einen Schlüssel in der Hand zum Verständnis nicht nur der alten Ägypter, sondern auch unseres eigenen Interesses an ihnen. Unser Streben, mit den Toten im Gespräch zu bleiben, führt uns immer weiter zurück, bis zu den Toten, die vor mehr als 5000 Jahren gelebt haben.

Zum Schluss möchte ich auf eine ganz andere ägyptische Idee der Verbindung von Tod und Zeit eingehen, die allem radikal widerspricht, was ich bisher entwickelt habe. Diese ketzerische Gegenstimme hat einen besonderen Ort in der ägyptischen Lebenswelt: das Fest. Man sollte meinen, dass beim festlichen Gelage Tod und Vergänglichkeit vollkommen ausgeblendet sind, aber genau das Gegenteil ist der Fall.

Der griechische Historiker Herodot, der Ägypten um 450 v. Chr. bereiste, berichtet von einer eigentümlichen Festsitte:

„Beim Gastmahl, wie es die Reichen halten, trägt nach der Tafel ein Mann ein hölzernes Bild einer Leiche, in einem Sarge liegend, herum. Es ist aufs beste geformt und bemalt und ein oder zwei Ellen lang. Er hält es jedem Zechgenossen vor und sagt: ,den schau an und trink und sei fröhlich! Wenn du tot bist, wirst du, was er ist"." (II Kap. 78.)

Das klingt wie die genaue Umkehrung der Lebensmaxime des Vezirs AmunUser, der meinte, das diesseitige Leben gering achten zu sollen angesichts der Ewigkeit des Jenseits. Gerade weil das Leben so kurz und vergänglich ist, muss man es zum Fest machen und genießen. Im Gastmahl des Trimalchio (Kap. 34) des Petronius wird eine ähnliche Sitte berichtet. Dort wird zum hundertjährigen Falernerwein ein silbernes Skelett aufgetragen. $\mathrm{Zu}$ dieser 
römischen Sitte gehören auch die ,Skelettbecher', die dem Zecher mit dem Emblem seiner Vergänglichkeit zugleich auch die Devise ktô chrô („Erwirb und genieße") vor Augen führen. Damit ist gemeint, dass das Leben sich nicht in bloßem Erwerbsstreben erschöpfen darf, sondern auch dem Genuss Raum geben soll. Auf diese Maxime stößt man auch im Alten Ägypten. So lehrt es auch der weise Ptahhotep, der Verfasser der berühmtesten ägyptischen Lebenslehre:

„Folge deinem Herzen, solange du lebst, und tu nicht mehr, als geboten ist.

Verringere nicht die Zeit des Dem-Herzen-Folgens!

Abscheu des $\mathrm{Ka}$ ist es, ihm seine Zeit zu zerstören.

Betreibe nicht die Tagesgeschäfte

über das, was nötig ist, dein Haus zu bestellen.

Auch der Besitz dessen wächst, der seinem Herzen folgt,

aber nichts nützen Reichtümer, wenn das Herz vernachlässigt wird.“

Das ist ein Plädoyer für die Muße im Zeithaushalt des vornehmen Ägypters. Es ist dieselbe Weisheit, die auch mit den zwei Wörtern auf den römischen Skelettbechern ausgedrückt ist: ktô chrô, „Erwirb und genieße“. Die Muße ist der Ort der Schönheit im ägyptischen Leben; sie dient der Belebung des Herzens, das im Betrieb der Alltagsgeschäfte verkümmert. Auch in Ägypten gilt die Devise, nicht nur zu erwerben, sondern auch zu genießen. Damit ist das Fest, der ,schöne Tag' gemeint, den man mit Familie und Freunden im festlichen Gelage verbringt, wie es die Gräber darstellen.

Zum ägyptischen Fest gehört ein Harfenspieler, der die Gäste mit einem Lied erfreut. In diesen Liedern ist nun sehr prominent von Tod und Vergänglichkeit die Rede:

„Das Lied, das im Hause [König] Antefs, des Seligen, steht, vor dem [Bilde des] Sänger[s] zur Harfe.

Glücklich ist dieser gute Fürst, nachdem das gute Geschick eingetreten ist! Geschlechter vergehen, andere kommen [var. bestehen] seit der Zeit der Vorfahren.

Die Götter, die vordem entstanden, ruhen in ihren Pyramiden.

Die Edlen und Verklärten desgleichen

sind begraben in ihren Pyramiden.

Die da Häuser bauten - ihre Stätte ist nicht mehr was ist mit ihnen geschehen?

Ich habe die Worte gehört des Imhotep und Hordedef, deren Sprüche in aller Munde sind.

Wo sind ihre Stätten? Ihre Mauern sind verfallen, sie haben keinen Ort mehr als wären sie nie gewesen.

Keiner kommt von dort, von ihrem Ergehen zu berichten, ihren Bedürfnissen zu erzählen, unser Herz zu beruhigen, bis auch wir gelangen, wohin sie gegangen sind. 
Du aber erfreue dein Herz und denke nicht daran!

Gut ist es für dich, deinem Herzen zu folgen, solange du bist.

Tu Myrrhen auf dein Haupt,

kleide dich in weißes Leinen, salbe dich mit echtem Öl des Gotteskults, vermehre deine Schönheit, lass dein Herz dessen nicht müde werden!

Folge deinem Herzen in Gemeinschaft deiner Schönen, tu deine Dinge auf Erden, kränke dein Herz nicht, bis jener Tag der Totenklage zu dir kommt.

Der ,Müdherzige' hört ihr Schreien nicht, und ihre Klagen holen das Herz eines Mannes nicht aus der Unterwelt zurück.

Refrain: Feiere den schönen Tag, werde dessen nicht müde!

Bedenke: Niemand nimmt mit sich, woran er gehangen, niemand kehrt wieder, der einmal gegangen."

Diese Weisheit hätte man den Ägyptern nicht zugetraut. Sie gehört in Ägypten aber unabdingbar zur Idee des Festes, und nicht nur in Ägypten, sondern in der ganzen alten Welt.

Im Gilgamesch-Epos ist es die Schankwirtin Siduri, die dem Gilgamesch diese Weisheit vor Augen führt. Gilgamesch ist auf der Suche nach Unsterblichkeit bis ans Ende der Welt gelangt. Dort betreibt die Göttin Siduri eine Schenke und empfängt den erschöpften Helden mit derselben Botschaft wie der ägyptische Harfner:

„Gilgamesch, wohin läufst du?

Das Leben, das du suchst, wirst du nicht finden!

Als die Götter die Menschen erschufen, teilten den Tod sie der Menschheit zu, das Leben behielten sie für sich selbst.

$\mathrm{Du}$, Gilgamesch - dein Bauch sei voll, ergötzen magst du dich Tag und Nacht! Mache jeden Tag zum Fest!

Tanz und spiel bei Tag und bei Nacht!

Deine Kleidung sei rein, gewaschen dein Haupt, mit Wasser sollst du gebadet sein!

Schau den Kleinen an deiner Hand, beglücke die Gattin mit deiner Umarmung!

Solcherart ist, was den Menschen zu tun bleibt."

Selbst in der Bibel stoßen wir auf diese Weisheit. Dort ist es der Prediger, der das Lied des Festes und der Schönheit anstimmt:

„Auf? IB freudig dein Brot und trink vergnügt deinen Wein, denn das was du tust hat Gott längst so festgelegt, wie es ihm gefiel.

Trag jederzeit frische Kleider, und nie fehle duftendes Öl auf deinem Haupt. Mit einer Frau, die du liebst, genieß das Leben 
alle Tage deines eitlen Lebens.

Denn das ist dein Anteil am Leben und an dem Besitz, für den du dich anstrengst unter der Sonne.

Alles, was dir vor Handen kommt zu tun, das tue frisch: denn bei den Toten, dahin du fährst, ist weder Schaffen noch Planen, noch Erkenntnis und Weisheit mehr!“

Das Fest und die Freude an der Gattin im Arm und den Kindern an der Hand ist der Anteil des Menschen, sein Trostpreis für den Entzug des ewigen Lebens, das sich die Götter vorbehalten haben. Was den Menschen bleibt, denen die Unsterblichkeit vorenthalten ist, ist die Erhöhung des Diesseits durch Schönheit und Liebe.

Diese resignative Einstellung zu Tod und Zeit passt zu Kulturen, die das Ideal der Unsterblichkeit nicht kennen und davon ausgehen, dass der Mensch nach dem Tod in ein Schattenreich übergeht, wo er nichts als tot ist. In einer Kultur wie der ägyptischen, die von der Verheißung der Rechtfertigung und Unsterblichkeit bestimmt ist, überrascht sie dagegen. Auch die Ägypter selbst haben den Widerspruch empfunden, den diese Lieder zur offiziellen Lehre darstellen. So beginnt ein anderes Harfnerlied:

„Ich habe diese Lieder gehört, die in den Gräbern der Vorfahren stehen und was sie erzählen zur Erhöhung des Diesseits und zur Herabsetzung des Jenseits.

Warum wird dergleichen angetan dem Lande der Ewigkeit?“

„Erhöhung des Diesseits“ - darum geht es, und gerade nicht darum, alles auf die Ewigkeit zu setzen, die man im Grabe verbringt, und darüber das „Wenige an Leben“ zu vergessen, das man auf Erden verbringt. „Was man von der Minute ausgeschlagen“, heißt es in Schillers Gedicht „Resignation“, „gibt keine Ewigkeit zurück.“

Nicht nur in den Festliedern, sondern auch in einem Literaturwerk hören wir diese Gegenstimme zu dem, was den Ägyptern doch das Heiligste und Wichtigste war. Das ist ein Streitgespräch zwischen einem ,Ich` und seinem ,Ba‘ (was wir mit „Seele“ übersetzen). Das Ich denkt ganz in den Kategorien der Resultativität und malt seinem $\mathrm{Ba}$ die Verheißungen eines guten Todes aus, nachdem für die Fortdauer im „schönen Westen“ Vorsorge getroffen wurde: durch Anlage eines Grabes und Bestellung eines Erben. Ihm ist die Zeit des ,solange etwas ist“ unerträglich geworden, und er sehnt sich nach der ruhigen Fortdauer dessen, was es gewesen sein wird.

Dem hält nun der $B a$ in schonungslosester Weise den illusionären Charakter der resultativen Dauer entgegen, die das Ich durch Anlage eines steinernen Grabes und den Vollzug der Totenriten realisieren zu können glaubt:

„Wenn du an das Begräbnis denkst: Ein Herzensjammer ist das, ein Hervorholen der Tränen ist das durch das Traurigmachen eines Menschen, das Herausholen eines Menschen ist das aus seinem Haus, um ihn in die Wüste zu werfen. 
Du kannst nicht wieder herauskommen, die Sonnen zu sehen.

Die da bauten in Granit,

die Kapellen anlegten in schönen Pyramiden,

in vollendeter Arbeit,

wenn ihre Erbauer zu Göttern geworden sind,

blieben ihre Opfersteine leer wie die Müden,

die am Uferdamm gestorben sind aus Mangel an einem Hinterbliebenen.

Das Wasser hat sich seinen Teil genommen, die Sonnenglut desgleichen,

die Fische des Ufers reden mit ihnen.

Höre du auf mich! Hören ist für die Menschen gut.

Folge dem schönen Tag! Vergiss die Sorge!“”

Dass ein Text eine so extreme Gegenstimme zu Wort kommen zu lassen und eine solche semantische Spannung aufzubauen vermag, zeigt die Größe und Tiefe der ägyptischen Literatur, ihre Fähigkeit, die Fundamentalien der eigenen Kultur zu reflektieren. Im Gilgamesch-Epos und im Kohelet geht es um die Feststellung der Grenzen des Menschseins und die Etablierung eines Daseinsverständnisses, das der allgemeinen Grundausrichtung der mesopotamischen, altisraelitischen und griechischen Kultur entspricht. In den ägyptischen Festliedern und im Disput zwischen Ich und Ba dagegen mit ihrer Kritik des resultativen Denkens (,Vergiss die Sorge!“") und Aufforderung zum Genuss des Lebens, weil es einmalig, endlich und unfortsetzbar ist („Feiere den schönen Tag!“"), geht es um den Widerspruch gegen das allgemeine Daseinsverständnis. Im Literaturwerk des Papyrus Berlin 3024 ist dieser Widerspruch aufgehoben in dem größeren Zusammenhang des Gesprächs, in dessen Verlauf sich die beiden Kontrahenten zuletzt einigen. In den Festliedern ist es der Ausnahmecharakter des Festes als eines ,Heterotops', der diese Gegenstimme in einen größeren Zusammenhang einbettet. Gerade weil die Idee des Totengerichts und die Verheißung des ewigen Lebens im ägyptischen Denken so fest verwurzelt sind, kann für die Dauer des Festes die Gegenstimme zu Wort kommen, die das Diesseits erhöht und das Jenseits herabsetzt. Die Bibel lehrt uns, was Vielstimmigkeit ist und welches $\mathrm{Ma} \beta$ an Widersprüchen selbst in einer kanonisierten Tradition möglich ist. Im Alten Ägypten gab es weder Orthodoxie noch einen Kanon heiliger Schriften. So sollte uns auch hier kulturelle Vielstimmigkeit nicht allzu sehr verwundern. 